\title{
Effects of drying on total polyphenols content and antioxidant properties of Carica papaya leaves
}

\begin{abstract}
Background: Papaya is widely grown in Malaysia and normally only the fruits are consumed. Other parts of the plant such as leaves, roots, bark, peel, seeds and pulp are also known to have medicinal properties and have been used to treat various diseases. Papaya leaves also contain flavonoids, alkaloids phenolic compounds and cynogenetic compounds, and are also reported to be able to treat dengue fever. Results: Studies were carried out on drying of papaya leaves using hot air $\left(60,70\right.$ and $\left.80{ }^{\circ} \mathrm{C}\right)$, shade and freeze drying. Effective diffusivities were estimated ranging from $2.09 \times 10-12$ to $2.18 \times 10-12 \mathrm{~m} 2 \mathrm{~s}-1$ from hot air drying, which are within the order of magnitudes reported for most agricultural and food products. The activation energy to initiate drying showed a relatively low value $(2.11 \mathrm{~kJ}$ mol-1 $)$ as a result of the thin leave layer that eased moisture diffusion. In terms of total polyphenols content and antioxidant activities, freeze-dried sample showed a significantly higher $(\mathrm{P}<0.05)$ total polyphenols content $[2158 \mathrm{mg}$ gallic acid equivalent $100 \mathrm{~g}$ dry weight-1 ] and antioxidant activities [2,2'-azino-bis(3-ethylbenzothiazoline6-sulfonic acid) $($ ABTS $)=571 \mathrm{mg}$ TE $100 \mathrm{~g} \mathrm{DW}$-1 and 2,2'-diphenyl-1-picrylhydrazyl $(\mathrm{DPPH})=$ $215 \mu \mathrm{g} \mathrm{mg}-1$ ] compared to hot air and shade dried samples. Conclusion: Freeze dried sample retained the most total polyphenols content and showed the highest antioxidant activities in both ABTS and DPPH antioxidant assays. Hot air and shade drying are not conducive with repect to preserving the antioxidants as a result of possible thermal degradation at elevated temperatures and oxidations under prolonged drying condition.
\end{abstract}

Keyword: Carica papaya; Antioxidants; Diffusivity; Papaya leaves; Polyphenols 